\title{
Plurale Lehre in den \\ Wirtschaftswissenschaften an der \\ Georg-August-Universität Göttingen
}

\section{Anne Berner, Franziska Dorn, Christian Ochsner, Alexander Silbersdorff und Lukas Wolfinger}

\section{Zusammenfassung}

In diesem Beitrag zeigen wir auf, welche Entwicklungen in der wirtschaftswissenschaftlichen Lehre an der Universität Göttingen 2007 zur Gründung einer Hochschulgruppe führten und wie diese die Lehre an der Wirtschaftswissenschaftlichen Fakultät um eine pluralistische Perspektive ergänzt hat. Im Zuge dessen werden wesentliche Defizite der Lehrsituation aufgezeigt, das Kooperationsverhältnis mit der Fakultät erörtert und die gruppeninternen Prozesse, die der studentisch gestalteten Lehre zugrunde liegen, diskutiert. Wir legen am Beispiel von studentisch organisierten Seminaren und

Hochschulgruppe Kritische Wirtschaftswissenschaften Göttingen
A. Berner $(\bowtie) \cdot$ F. Dorn $\cdot$ C. Ochsner $\cdot$ A. Silbersdorff
Georg-August-Universität Göttingen, Göttingen, Deutschland
E-Mail: anne.berner@uni-goettingen.de
F. Dorn
E-Mail: fdorn@uni-goettingen.de
C. Ochsner
E-Mail: christian.ochsner@uni-goettingen.de

A. Silbersdorff

E-Mail: asilbersdorff@uni-goettingen.de

L. Wolfinger

Göttingen, Deutschland

E-Mail: wolfinger@sub.uni-goettingen.de 
Ringvorlesungen dar, unter welchen Bedingungen pluralistische Lehre einen Beitrag leisten kann, die Mängel in der Lehre zu beseitigen und wie dies auf die Hochschulgruppe und die Fakultät zurückwirkt..

\section{Schlüsselbegriffe}

Heterodoxie $\cdot$ Pluralismus $\cdot$ Hochschuldidaktik $\cdot$ VWL-Kritik ·

Selbstorganisiertes Lernen

\section{$1 \quad$ Entstehungskontext}

Das Leitbild der Universität Göttingen beschreibt das Ideal, dass die universitäre Gemeinschaft von ,intellektueller Neugier, konstruktiver, kritischer Diskussionskultur und der Partizipation in universitären und gesellschaftlichen Belangen“" lebt (Georg-August-Universität Göttingen 2019c). Dies erschien Studierenden im wirtschaftswissenschaftlichen Studium im Jahr 2007 nur unzulänglich gegeben, was die Gründung der Hochschulgruppe „Kritische Wirtschaftswissenschaften Göttingen" zur Folge hatte. Ziel der Gruppe war und ist die kritisch-reflektierende Auseinandersetzung mit den Inhalten der wirtschaftswissenschaftlichen Lehre und Forschung sowie die konstruktive Ergänzung der Lehre um Inhalte der pluralen Ökonomie an der Georg-August-Universität Göttingen. Die Wahrnehmung der Hochschulgruppe wird durch verschiedene Studien gestützt. Sie stellen fest, dass Studierende der Wirtschaftswissenschaften sich von den realökonomischen Geschehnissen und Problemen durch ihr Studium abgeschnitten und auf eine Lösung derselben nicht vorbereitet fühlen. Sie fordern eine größere Diversität an ökonomischen Theorien in der Lehre (Green 2013; Pühringer und Bäuerle 2019; Engartner und Schweitzer-Krah 2019).

Die Hochschulgruppe hat zu Themen aus der pluralen Ökonomie zahlreiche universitäre und außeruniversitäre Veranstaltungen organisiert. Seit ihrer Gründung wurden zudem zwei studentisch organisierte Lehrformate - das autonome Seminar und die Ringvorlesung „Heterodoxie in der VWL“ - in den Wahlbereich des wirtschaftswissenschaftlichen Curriculums der Universität Göttingen aufgenommen. Im Rahmen der Konzeption und Durchführung dieser Veranstaltungen hat sich die Gruppe intensiv mit unterschiedlichen Lehr-, Lern- und Prüfungsformen auseinandergesetzt. Die dabei gewonnenen Erkenntnisse sollen im folgenden Beitrag kritisch reflektiert und festgehalten werden. 
$\mathrm{Zu}$ diesem Zweck wollen wir in einem ersten Schritt zunächst die lokalen Umstände skizzieren, die sowohl die Entwicklung als auch die Durchführung der studentisch organisierten Lehrformate bedingt und ermöglicht haben. Sodann werden wir in zwei weiteren Abschnitten diskutieren, wie die weitgehend autonome Organisation der Lehrveranstaltungen und deren Integration in das universitäre Curriculum didaktisch produktive Gruppenprozesse fördern und formen. In einem Resümee fassen wir unsere Ergebnisse schließlich zusammen.

\subsection{Kritik an der wirtschaftswissenschaftlichen Lehre}

Die Lehre an der Wirtschaftswissenschaftlichen Fakultät Göttingen erschien (und erscheint in Teilen weiterhin) trotz engagierter Dozentinnen und Dozenten durch fünf zentrale Defizite charakterisiert: Sie ist 1) methodisch und inhaltlich unvollständig. Zudem bietet sie 2) zu selten und in zu geringem Umfang die Möglichkeit zur kritischen Diskussion. Des Weiteren fördert oder fordert sie 3) die selbstständige Lektüre und Reflexion von Forschungsbeiträgen nur unzureichend. Darüber hinaus ist sie 4) weitgehend ahistorisch sowohl im Hinblick auf die Ideengeschichte als auch im Hinblick auf die Geschichte wirtschaftlicher Entwicklung. Schließlich dominieren 5) als Prüfungsformen unter Zeitdruck zu lösende mathematische Aufgaben und die Abfrage von auswendig gelerntem Wissen, wohingegen die gedankliche Eigenständigkeit bei der Lösung wirtschaftlicher Fragen weder besonders gefördert noch gefordert wird. Diese fünf Kritikpunkte werden im Folgenden etwas eingehender erläutert und belegt:

$\mathrm{Zu}$ Kritikpunkt 1): Die Unvollständigkeit der Lehre sei an dieser Stelle nur kurz exemplarisch an der VWL verdeutlicht: Zum einen wird methodisch bis dato der abstrakt-mathematische Zugang der Rational-Choice Modellierung im Zusammenspiel mit rein quantitativer Empirie gelehrt. Zum anderen beschränkt sich der Inhalt der Lehre - vor allem im Bachelor - auf Gleichgewichtsmodelle (in der Mikroökonomik und deren Anwendungen) und auf die neoklassisch-keynesianische Synthese bzw. neoklassische Wachstumstheorie (in der Makroökonomik). ,Reflexive' Lehrinhalte, wie Wissenschaftstheorie, Wirtschaftsgeschichte oder Wirtschaftsethik sind in Göttingen hingegen aktuell kaum vorhanden, was sich auch im 41. Platz im entsprechenden Ranking von Fauser und Kaskel (2016) in dieser Kategorie widerspiegelt. Eine Untersuchung der Modulbeschreibungen der Universität Göttingen von Beckenbach et al. (2016) ergab, dass in der Beschreibung der Mikroökonomie ausschließlich als orthodox kategorisierte Begriffe (insbesondere „Gleichgewicht“, „Marktversagen“ oder „Rationalität“) aufgeführt werden. Auffällig ist jedoch, dass im Gegensatz dazu 
in den makroökonomischen Modulbeschreibungen die Nennung von heterodoxen (insbesondere „Kritik“, „Kapitalakkumulation“, „Modellvielfalt“ und „Krise“) und orthodoxen (insbesondere „Gleichgewicht", „IS-LM" und „AS-AD“) Begriffen auf einem relativ hohen Niveau ausgeglichen ist (Beckenbach et al. 2016).

Zu den Kritikpunkten 2) und 3): Da die wirtschaftswissenschaftliche Lehre überwiegend in Vorlesungen mit begleitenden Übungen erfolgt, in denen vorrangig vorgegebene Inhalte erarbeitet werden, erhalten Studierende vergleichsweise selten die Möglichkeit zu kritischer Reflexion und Diskussion. Die Hauptseminare, die nur in geringem Umfang vorgeschrieben sind und so gut wie immer als Blockveranstaltung stattfinden, bestehen überwiegend aus Referaten der Teilnehmenden. Dies ist prinzipiell zu begrüßen, allerdings werden die Vorträge kaum diskutiert und insgesamt bleibt auch in den Seminaren nur wenig Spielraum für eigene Gedanken der Studierenden. Diese sind es daher nicht gewohnt, Inhalte durch Textdiskussion zu erschließen, Argumentationsgänge nachzuzeichnen und im Hinblick auf ihre Stringenz und Validität zu beurteilen. Eine weitere Konsequenz der beschriebenen Lehrorganisation ist, dass Studierende erst vergleichsweise spät in Kontakt mit Forschungspublikationen kommen, da sie - speziell in den ersten zwei Studienjahren - fast ausschließlich mit Lehrbüchern konfrontiert sind, die wenig Vielfalt aufweisen (van Treeck und Urban 2017). Dies unterstreicht auch eine Befragung von Studierenden des vierten Fachsemesters aus fünf großen Universitätsstädten: $75 \%$ der Befragten waren der Meinung, dass Wissen aus angrenzenden Sozialwissenschaften zum Verständnis ökonomischer Sachverhalte notwendig ist, dieser interdisziplinäre Zugang jedoch (zu) selten stattfindet (Engartner und Schweitzer-Krah 2019).

$\mathrm{Zu}$ Kritikpunkt (4): Die Lehre in Göttingen verortet wirtschaftswissenschaftliche Theorien und Modelle selten in ihrem historischen Kontext, weder im Hinblick auf die Phänomene, die zu ihrer Entstehung führten, noch im Hinblick auf die Debatten, aus denen sie hervorgingen. Sie folgt damit freilich nur den allgemeinen Trends, denn wie Graupe (2017) in ihrer Analyse der - auch in Göttingen verwendeten - Standardlehrbücher der Ökonomie zeigt, werden in denselben z. B. die ,geistes- und ideengeschichtlichen Hintergründe [...] der verwendeten mechanistischen Konzepte und Metaphern" verschwiegen (Graupe 2017, S. 87). Dies ist keine optimale Vorbereitung auf die Tätigkeit in wirtschaftswissenschaftlicher Forschung.

$\mathrm{Zu}$ Kritikpunkt (5): Im Vergleich zu anderen Sozialwissenschaften und den Geisteswissenschaften ist es in der wirtschaftswissenschaftlichen Lehre in Göttingen unüblich, von den Studierenden die Formulierung und Verteidigung 
eigener Gedanken in Auseinandersetzung mit dem aktuellen Forschungsstand zu verlangen. Stattdessen wird mithilfe von Klausuren geprüft. Mittelwege wie Projektarbeiten sind nur im Masterstudium verbreitet (Georg-August-Universität Göttingen 2019a, b).

Die Summe dieser Unzulänglichkeiten in der Lehre führte (und führt) bei den Mitgliedern der Hochschulgruppe zu Unverständnis und Unzufriedenheit. Ihnen fehlte der Bezug zwischen der, neoklassisch“ geprägten Lehre und den wirtschaftlichen Fragen und Problemen, die sie zu ihrer Studienwahl motiviert hatten. Aus diesem Grund wurde im Wintersemester 2007/2008 unter dem Eindruck der Finanzkrise ein Lesekreis von interessierten Studierenden eingerichtet, aus dem die Hochschulgruppe hervorging. Diese hat den Anspruch, die Lehre an der Wirtschaftswissenschaftlichen Fakultät der Universität Göttingen konstruktiv mitzugestalten und dabei die oben aufgeführten Kritikpunkte zu adressieren. Die Mitglieder der Hochschulgruppe etablierten bereits im Jahr 2010 ein autonomes Seminar, das im Wahlbereich verankert ist. Zudem wird seit 2014 jährlich eine Ringvorlesung organisiert. Um den Entstehungskontext der Veranstaltungen besser verorten zu können, wird im Folgenden die Einbindung der Veranstaltungen an der Universität und die Zusammenarbeit mit der Fakultät erläutert.

\subsection{Einbindung an der Universität}

Für beide Lehrveranstaltungen gilt, dass die Wirtschaftswissenschaftliche Fakultät - nach kritischer Prüfung - der Hochschulgruppe die Möglichkeit eröffnet hat, das jeweilige Format frei zu konzipieren und durchzuführen. Dies war nicht zuletzt aufgrund der 2007 eingeführten Studiengebühren möglich, denn durch die ausschließlich für die Lehre vorgesehenen Mittel konnten studentische Hilfskraftstellen, Stellen für wissenschaftliche Mitarbeiterinnen und Mitarbeiter sowie Vortragshonorare bezahlt werden. Seit Abschaffung der Studiengebühren in Niedersachsen können die Stellen dank Studienqualitätsmitteln bis jetzt weiterhin finanziert werden. Die Unterstützung und die Anrechnung der Lehrformate im Wahlbereich der wirtschaftswissenschaftlichen Studiengänge war anfänglich vor allem einem der Göttinger VWL-Professoren aus dem Bereich der Entwicklungsökonomie zu verdanken. Beide Lehrformate wurden zunächst offiziell durch diesen Lehrstuhl angeboten. Mittlerweile erfahren die Veranstaltungen und das Engagement der Hochschulgruppe jedoch von einer Vielzahl von Professuren 
dankenswerte Unterstützung ${ }^{1}$. Diese Akzeptanz konnte vor allem erreicht werden, indem die Mitglieder der Hochschulgruppe stets an einem konstruktiven Dialog mit den Lehrverantwortlichen der Fakultät interessiert waren und sind: Professorinnen und Professoren sowie Dozentinnen und Dozenten wurden zu Diskussionen auf das „Bunte Sofa“ der Gruppe, zur Teilnahme an Podien oder Vorträgen eingeladen. Sobald die Lehrenden das Interesse und Engagement der Mitglieder der Hochschulgruppe erkannten, für die die kritisch-wissenschaftliche Reflexion im Vordergrund steht und die nicht zum Ziel haben, das Lehrpersonal zu ,kritisieren “ oder mit Lesekreisen die Hürden der Mathematik zu umgehen, war meist sehr schnell eine gute Kommunikationsbasis vorhanden - insbesondere, da eine Reihe von Dozentinnen und Dozenten mit der Verschulung des Studiums, die das Bachelor- und Mastersystem mit sich gebracht hat, selbst unzufrieden ist. Da der Lehre der Hochschulgruppe nach vereinzelten Besuchen der Dozierenden viel Vertrauen entgegengebracht wird, können alle Veranstaltungen der Ringvorlesung und des autonomen Seminars ohne weitere Einwände der betreuenden Dozierenden stattfinden. Jeder Durchgang wird inhaltlich mit der Fakultät abgestimmt, de facto gab es nie Einwände oder Vorbehalte seitens der Fakultät.

\section{Innovative Lösung im Sinne einer pluralen, sozioökonomischen Hochschulbildung}

Die Hochschulgruppe hat in den letzten Jahren mehrere Ansätze in der Lehre erprobt, um Studierende für eine kritische Auseinandersetzung mit ihrem Fach zu begeistern und die im vorangegangenen Kapitel aufgeführten Kritikpunkte innovativ zu adressieren.

Die plurale Lehre in Göttingen richtet sich vornehmlich an Studierende der Wirtschaftswissenschaftlichen Fakultät. Die Mitglieder der Hochschulgruppe sowie die Teilnehmerinnen und Teilnehmer sind mehrheitlich Studierende der VWL, die oft noch ein weiteres Fach studieren. Diese Fokussierung auf eine Zielgruppe schließt jedoch Studierende anderer Fächer nicht aus. Bei den Lehrveranstaltungen besteht keine Anwesenheitspflicht und auch keine Pflicht, eine

\footnotetext{
${ }^{1}$ Neben der Professur für Entwicklungsökonomik bringen sich insbesondere die Professur für Wirtschaftsgeschichte, die Professur für Wirtschaftspolitik und Mittelstandsforschung sowie die Professur für Statistik aktiv in die Lehre pluraler Ökonomik an der GeorgAugust-Universität Göttingen ein.
} 
Prüfung abzulegen. Wer ein besonderes Interesse an einem bestimmten Vortrag hat, ist nicht gebunden, die ganze Veranstaltung zu besuchen. Aufgrund der volkswirtschaftlichen Ausrichtung der Mehrheit der Mitglieder der Hochschulgruppe orientieren sich die Ringvorlesung und das Seminar daran, welche Inhalte im VWL-Studium wenig Beachtung finden sowie an den thematischen Wünschen der Studierenden. Beide Lehrformate sind als Module im Wahlbereich der wirtschaftswissenschaftlichen Studiengänge anrechenbar (Georg-August-Universität Göttingen 2019a). Ab dem Sommersemester 2020 wird die Ringvorlesung als Modul im Wahlpflichtbereich des Bachelorstudiums der Wirtschaftswissenschaftlichen Fakultät unter dem Titel „Ökonomische Perspektiven jenseits der Neoklassik" (Modulhandbuch ab SoSe 2020) verstetigt und als volkswirtschaftlicher Kernkurs akkreditiert. In den Masterprogrammen der Wirtschaftswissenschaftlichen Fakultät kann im Wahlbereich entweder das autonome Seminar oder die Ringvorlesung innerhalb des Moduls „Plurale Ökonomik“ eingebracht werden (Georg-August-Universität Göttingen 2019b).

\subsection{Autonomes Seminar}

Das Seminar ist in zweierlei Hinsicht, autonom': Erstens ist die konkrete Ausgestaltung der Seminarform nicht festgelegt (Georg-August-Universität Göttingen 2019a). In der Vergangenheit wurden verschiedene Formate erprobt. Dazu gehört beispielsweise der von den Teilnehmerinnen und Teilnehmern getragene Diskussionskreis, in dem die Studierenden selbst eine Seminareinheit vorbereiten und durchführen, wobei leitende Tutorinnen und Tutoren koordinierend zur Seite stehen. Ein alternatives Format ist die forschungsorientierte Vorbereitung auf eine Konferenz, bei der die Teilnehmerinnen und Teilnehmer gemeinsam einen Konferenzbeitrag erarbeiten - in Form eines Vortrags oder wissenschaftlicher Plakate zu den von ihnen erarbeiteten Themen. Sehr beliebt ist das geführte Lektüreseminar. In diesem Fall bereiten die Tutorinnen und Tutoren für jeden Termin einen wissenschaftlichen Text vor, der von den Teilnehmerinnen und Teilnehmern gelesen und im Seminar gemeinsam diskutiert wird. Durch diese Form der Autonomie kann ein Großteil der oben genannten und in der Forschung belegten Kritikpunkte (Green 2013; Pühringer und Bäuerle 2019; Engartner und Schweitzer-Krah 2019) vermieden werden: Die beschriebenen Formate eröffnen die Möglichkeit zu diskutieren, eigene Gedanken zu entwickeln und die Teilnehmenden mit Forschungsliteratur und unterschiedlichen Arten wissenschaftlicher Argumentation und Bewertung vertraut zu machen. 
Zweitens ist das Seminar in der inhaltlichen Gestaltung autonom. Es wird zwar von einer Dozentin oder einem Dozenten der Fakultät formal betreut und verantwortet, allerdings entscheidet die Hochschulgruppe (bzw. die organisierende Kleingruppe) über die inhaltliche Ausrichtung, die Seminarform und die Ausgestaltung. Das genaue Thema ${ }^{2}$ der jeweiligen Lehrveranstaltung variiert von Semester zu Semester und wird durch die organisierende Kleingruppe festgelegt. Dies ermöglicht es, Inhalte jenseits des Standard-Curriculums zu erarbeiten. So beschäftigen sich die Studierenden in den Seminaren mit Wissenschaftstheorie, den Annahmen ökonomischer Modellierung, Dogmengeschichte, der Finanzkrise, Machtbeziehungen oder ökonomischer und sozialer Ungleichheit.Die Zahl der Teilnehmenden in den Seminaren beträgt pro Semester ca. zehn bis 15 Personen. Hinzu kommt die organisierende Kleingruppe, die aus drei bis sieben Personen besteht. Die Prüfung wird von der begleitenden Dozentin oder dem begleitenden Dozenten abgenommen. Im Seminar handelt es sich dabei in der Regel um eine Hausarbeit zu einer eigens gewählten Fragestellung (GeorgAugust-Universität Göttingen Göttingen 2019a) ${ }^{3}$.

\section{$2.2 \quad$ Ringvorlesung}

Das Konzept der Ringvorlesung wurde nach dem Vorbild einer ähnlichen Veranstaltung der Hochschulgruppe „Plurale Ökonomie“ der Universität Bayreuth gestaltet und an die Möglichkeiten in Göttingen angepasst. Die Ringvorlesung findet in der Regel als Einführungsvorlesung zum Thema „Heterodoxie in der VWL“" statt. Alle zwei bis drei Durchgänge stehen jedoch auch spezifische Themen stärker im Fokus ${ }^{4}$, welche von der organisierenden Kleingruppe festgelegt werden. Diese entscheidet auch über die Einladung der Referentinnen und Referenten. Die Vortragenden werden sowohl aus dem heterodoxen als auch dem

\footnotetext{
${ }^{2}$ Es wurden bereits sieben Seminare mit den Themen: „Postwachstumsökonomie“, „Homo Oeconomicus“, „Ethik und Wirtschaft“, „Wirtschaftswachstum und Nachhaltigkeit: Geht das zusammen?“, „Macht und Ökonomie“, „Wirtschaftsnobelpreisträger und ihre Thesen“ und „Werte und Ethik in der VWL“ angeboten.

${ }^{3}$ Diese Prüfungsform kann variieren. In der Vergangenheit gab es neben Hausarbeiten auch die Möglichkeit ein Poster für eine Konferenz zu gestalten und dieses dort vorzustellen.

${ }^{4}$ Ausgewählte Themen der Ringvorlesung waren „Arbeit, Lohn und Einkommensverteilung", „Current Research Topics in Heterodox Economics" und „Sharing, Caring, Cooperation".
} 
orthodoxen Spektrum der Wirtschaftswissenschaften ausgesucht; sie stammen aber ebenso aus verwandten Geistes- und Sozialwissenschaften oder tragen mit politischem, aktivistischem oder journalistischem Hintergrund ihre Perspektive bei. Bewusst wird ein Drittel der Vorlesungszeit für Diskussion reserviert und die Studierenden werden motiviert, die Vortragenden mit Fragen herauszufordern. Die Ringvorlesung lässt den Studierenden der Hochschulgruppe zwar viele Freiheiten, sie wird aber, anders als das autonome Seminar, durch wissenschaftliche Mitarbeiterinnen und Mitarbeiter betreut. Bisher sind das ehemalige Mitglieder der Hochschulgruppe, die inzwischen graduiert und an Lehrstühlen der Universität beschäftigt sind.

Begleitet wird die Veranstaltung von wöchentlichen Tutorien, in denen die Studierenden gehalten sind, den Vortrag der Woche in Diskussionsrunden oder Kleingruppen eingehender zu kontextualisieren und zu reflektieren. Ziel der Tutorien ist es insbesondere auch, eine Diskussionskultur $\mathrm{zu}$ etablieren, wozu verschiedene Methoden angewendet werden. Da bei den Vorträgen auch ökonomische Theorien behandelt oder angeschnitten werden, die in der ökonomischen Lehre in Göttingen nicht oder nur am Rande behandelt werden, geht es in den Tutorien oft auch darum, Konzepte zu klären sowie Annahmen zu hinterfragen. Uns ist dabei wichtig, sowohl die im Mainstream der ökonomischen Lehre vertretenen Theorien als auch die heterodoxen Ansätze kritisch zu betrachten. Um in den Tutorien Theorien und ihre Prämissen zu diskutieren, werden im Vorfeld einführende Texte an die Studierenden ausgegeben, die für das Tutorium vorbereitet und dort besprochen werden.

Abgerundet wird die Ringvorlesung durch eine Podiumsdiskussion am Ende des Semesters, wobei das Thema durch die Studierenden in der ersten Vorlesung mittels einer ,Papierfliegerwahl' bestimmt wird. Dabei schreiben alle Teilnehmenden ihre Themenwünsche auf Papierflieger, die dann gemeinsam nach vorne geworfen werden - eine Methode, die sich als ein effektiver ,Eisbrecher" bewährt hat.

Die Ringvorlesung belegen im Schnitt 50 Personen pro Semester. Die Zahl der Anwesenden unterliegt jedoch sowohl im Semesterverlauf als auch über die Semester hinweg teils starken Schwankungen. Die Mehrheit der Teilnehmenden sind Bachelorstudierende (siehe Abb. 1).

Die Prüfung in der Ringvorlesung erfolgt in Form einer elektronischen Klausur. Dieses Format ermöglicht uns - neben einem gut leserlichen Schriftbild bei den zu verfassenden Essays - eine Zeichenbegrenzung anstelle der sonst üblichen strikten Zeitbegrenzung. Die Studierenden haben so die Möglichkeit, in Form eines Essays ihre Gedanken zu strukturieren und auszuformulieren und sich ohne Zeitdruck kritisch mit einer aktuellen ökonomischen Problemstellung 


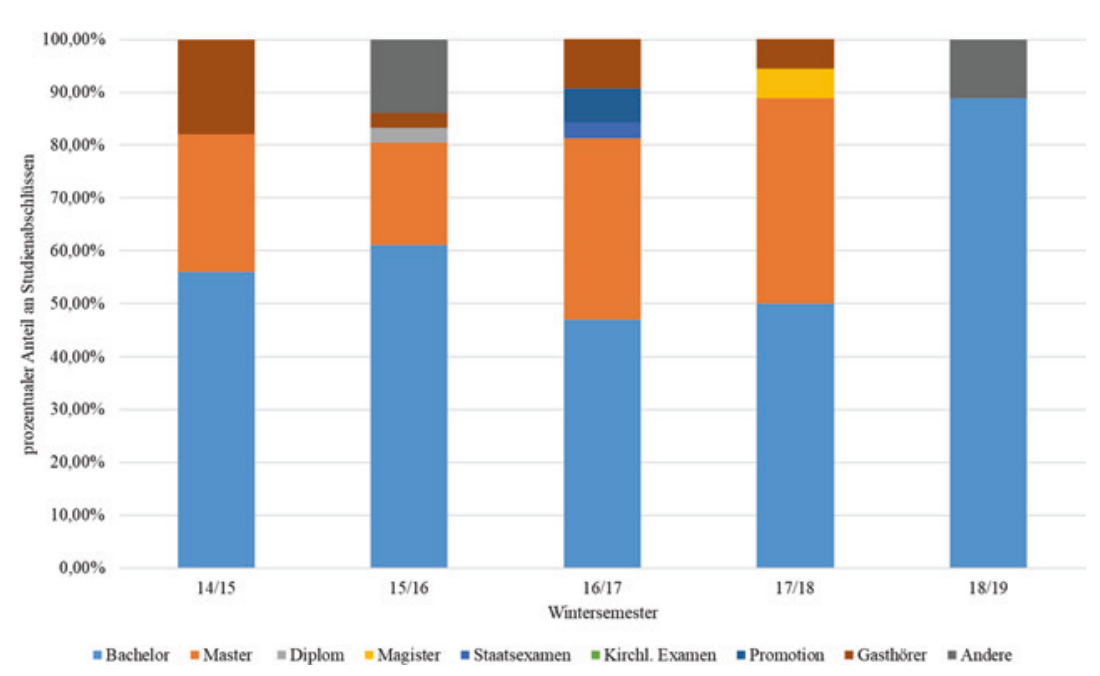

Abb. 1 Anteile der Abschlüsse ab dem WiSe 14/15 (eigene Auswertung)

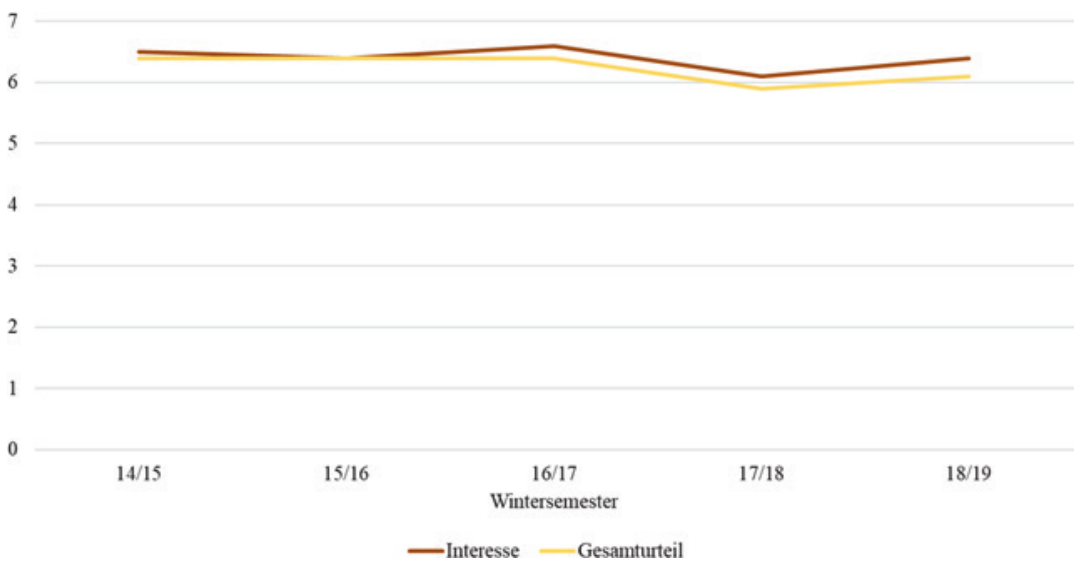

Abb. 2 Interesse der Studierenden im Vergleich zum Gesamturteil (eigene Auswertung)

auseinanderzusetzen. Die Auswertung unserer inoffiziellen Befragung zur Klausur zeigt, dass ein Großteil der Teilnehmenden diese Klausurform befürwortet. Die Studierenden zeigen ein hohes Interesse an der Veranstaltung, die sie über die Jahre konstant mit über sechs von sieben möglichen Punkten 
bewerten. Ebenfalls fällt das Gesamturteil, das sich je nach Semester bisher in einer Bandbreite zwischen 5,9 und 6,4 Punkten bewegte, sehr gut aus (siehe Abb. 2).

\section{$3 \quad$ Folgen/Wirkungen}

Die Folgen der durch die Hochschulgruppe angebotenen Lehrformate sind vielschichtig. Auf der einen Seite wird das curriculare Angebot der Universität erweitert und die Nachfrage von Studierenden nach inhaltlich und didaktisch alternativen Angeboten bedient. Auf der anderen Seite ist zur Realisierung dieses Angebots ein hoher Arbeitsaufwand aufseiten der durchführenden Personen zu verzeichnen.

\subsection{Folgen und Wirkungen in der universitären Lehre}

Wir legen in unseren Seminaren und Tutorien den Fokus auf Themen, die im regulären Lehrplan zu kurz oder gar nicht vorkommen. Aus diesem Grund ist eine der Hauptaufgaben der Gruppe der Tutorinnen und Tutoren, den Inhalt zu erarbeiten. Gerade die Vorbereitung einer Literaturliste, die dem heterodoxen und interdisziplinären Anspruch der Veranstaltungen genügt, benötigt vergleichsweise viel Zeit. So erfordert eine durchschnittliche Lehrveranstaltung im Vorlauf und während des Semesters in der Regel ein Engagement von ca. fünf bis acht Stunden pro Woche von zwei bis drei Personen, sodass die Betreffenden in der Regel kaum Zeit für weitere Aktivitäten der Hochschulgruppe haben. Eine weitere Folge des hohen gemeinsamen Arbeitsaufwands ist eine starke Identifikation und Auseinandersetzung der Gruppe der Tutorinnen und Tutoren mit den Themen des Seminars und vielfach auch eine deutliche Emanzipation von den vorgegebenen Lerninhalten des Standard-Curriculums.

Die genannten Formate führen (teilweise) auch zum Aufbrechen der klassischen Dozierenden-Studierenden-Dichotomie. Hinsichtlich der Anforderungen an die Tutorinnen und Tutoren wurde bewusst ein niedrigschwelliger Zugang ermöglicht, sodass auch Bachelorstudierende $\mathrm{zu}$ Beginn ihres Studiums Lehrtätigkeiten wahrnehmen konnten. Deshalb stellte sich oft die Frage, welche Rolle die Tutorinnen und Tutoren gegenüber den anderen Studierenden einnehmen können und sollen. Diesbezüglich wurde explizit ein Weg der möglichst flachen Hierarchien gewählt, sodass sich die Tutorinnen und Tutoren nicht als Expertinnen und Experten präsentierten, sondern vielmehr 
als studentische Teilnehmerinnen und Teilnehmer, die ggf. moderierende Aufgaben übernahmen, aber anderweitig keine exponierte Stellung in den Lehrveranstaltungen einnahmen.

\subsection{Folgen und Wirkungen für die durchführende Hochschulgruppe}

Die Erfahrung zeigt, dass die Gruppen der Tutorinnen und Tutoren, die diese Veranstaltungen organisieren, zum Zusammenhalt der Hochschulgruppe äußerst positiv beitragen. Zum einen geben sie der Gruppe universitätsöffentlich ein Gesicht und zum anderen tragen sie häufig die Themen der Lehrveranstaltung in die gruppeninternen Diskussionen. Umgekehrt ist festzuhalten, dass die Arbeit solcher Kleingruppen keine nachteiligen Effekte auf andere Aktivitäten der Hochschulgruppe hat bzw. hatte, da in Göttingen eine ausreichend große Basis an aktiven Studierenden vorhanden ist.

Zuletzt ist neben den hochschulinternen Folgen auch von Bedeutung, dass die Arbeit der Hochschulgruppe über die Universität hinaus Beachtung gefunden hat und stadtöffentlich eine Reihe von Diskursen zu ökonomischen Themen mitprägt - so wurden beispielsweise auf Grundlage von unseren Lehrveranstaltungen und unter Mithilfe von weiteren Gruppenmitgliedern öffentliche Podiumsdiskussionen mit mehreren hundert Zuschauerinnen und Zuschauern sowie mit einem breiten Medienecho zu verschiedenen Themen veranstaltet. Diese Außenwirkung der Hochschulgruppe wiederum führte zu einem hohen Zuspruch vonseiten der Fakultät - was wiederum Grundlage dafür war, dass wir kontinuierlich vonseiten des Dekanats und der Studierendenschaft Unterstützung erfahren.

Eine Auf- bzw. Übernahme der inhaltlichen wie didaktischen Anregungen, die vonseiten der Studierenden wie der Hochschulgruppe kommen, in das Lehrangebot ist bei den Professorinnen und Professoren der Fakultät bislang jedoch nur vereinzelt zu beobachten. Es stellt sich deshalb auch die Frage, inwieweit wir durch unsere eigenen Veranstaltungen dazu beitragen, Desiderata in der Lehre aufzuzeigen und Probleme zu beheben (wie in Abschn. 2.1 thematisiert) oder ob wir durch unsere Arbeit vielleicht sogar dazu beitragen, den Status quo zu stabilisieren. Die Diskussion über diese Frage dauert an. 


\section{Schlussbemerkungen}

Die Hochschulgruppe hat sich zum Ziel gesetzt, die kritische Auseinandersetzung mit den Lehrinhalten der Wirtschaftswissenschaften zu fördern und die Lehre in Göttingen um plurale Inhalte zu ergänzen. Die selbstständig durchgeführte Lehre ermöglichte den Teilnehmerinnen und Teilnehmern ebenso wie den die Lehrveranstaltungen betreuenden Tutorinnen und Tutoren eine intensive Auseinandersetzung mit Themen jenseits des Standard-Curriculums der VWL in einer Veranstaltung mit flachen Hierarchien und offener Diskussionskultur. Aufgrund der intrinsischen Motivation der Tutorinnen und Tutoren werden die Lehrinhalte begeistert und mithilfe interaktiver Methoden an die Teilnehmerinnen und Teilnehmer weitergegeben. Sowohl das autonome Seminar als auch die Ringvorlesung sind im Wahlbereich der wirtschaftswissenschaftlichen Studiengänge anrechenbar, wobei letztere Veranstaltung ab dem kommenden Semester in den Wahlpflichtbereich des VWL-Studiums integriert wird. Wesentliche Grundlage unserer Tätigkeit ist die Zusammenarbeit mit der Wirtschaftswissenschaftlichen Fakultät und eine aus unserer Sicht wechselseitig bestehende Wertschätzung.

Die Vorbereitung der beiden Lehrformate ist mit einem hohen personellen Aufwand für die Hochschulgruppe verbunden. Dieser Aufwand wird allerdings durch die positiven Effekte der Gruppenbildung und der stets wachsenden inhaltlichen, organisatorischen und didaktischen Fähigkeiten der Hochschulgruppe hinreichend kompensiert und ist somit in unserem Kontext gerechtfertigt. Wir glauben und hoffen, dass es an einer Vielzahl von deutschen Universitäten möglich ist und ggf. auch zum Standard wird, dass von Studierenden initiierte, plurale Veranstaltungen die dortigen Curricula des VWL-Studiums ergänzen und die Lehre kritisch und konstruktiv mitgestalten!

\section{Literatur}

Beckenbach, F., Daskalakis, M., \& Hofmann, D. (2016). Zur Pluralität der volkswirtschaftlichen Lehre in Deutschland. Eine empirische Untersuchung des Lehrangebots in den Grundlagenfächern und der Einstellung der Lehrenden. Marburg: Metropolis.

Engartner, T., \& Schweitzer-Krah, E. (2019). Students “ perception of the pluralism debate in economics: Evidence from a quantitative survey among German universities. International Review of Economics Education, 30, 1-12.

Fauser, H., \& Kaskel, M. (2016). Pluralism in economics teaching in Germany - evidence from a new dataset. Düsseldorf: Hans-Böckler-Foundation. https://www.boeckler.de/ pdf/v_2016_10_21_fauser.pdf. Zugegriffen: 15. Nov. 2019. 
Georg-August-Universität Göttingen (2019a). Modulverzeichnis- Bachelor-Studiengänge der Wirtschaftswissenschaftlichen Fakultät WiSe 19/20. Georg-August-Universität Göttingen. https://www.uni-goettingen.de/de/29209.html. Zugegriffen: 15. Nov. 2019.

Georg-August-Universität Göttingen (2019b). Modulverzeichnis- Master-Studiengänge der Wirtschaftswissenschaftlichen Fakultät WiSe 19/20. Georg-August-Universität Göttingen. http://www.uni-goettingen.de/de/29210.html. Zugegriffen: 15. Nov. 2019.

Georg-August-Universität Göttingen (2019c). Leitbild für das Lehren und Lernen. GeorgAugust-Universität Göttingen. https://www.uni-goettingen.de/de/leitbild+f\%C3\% BCr+ das+lehren+und+lernen/594258.html. Zugegriffen: 15. Nov. 2019.

Graupe, S. (2017). Beeinflussung und Manipulation in der ökonomischen Bildung. FGW-Studie Neues ökonomisches Denken 05. Düsseldorf: Forschungsinstitut für Gesellschaftliche Weiterentwicklung (FGW). http://www.fgw-nrw.de/fileadmin/user_ upload/NOED-Studie-05-Graupe-A1-komplett-Web.pdf. Zugegriffen: 15. Nov. 2019.

Green, T. L. (2013). Teaching (un)sustainability? University sustainability commitments and student experiences of introductory economics. Ecological Economics, 94, 135142 .

Pühringer, S., \& Bäuerle, L. (2019). What economics education is missing: the real world. International Journal of Social Economics, 46(8), 977-991.

van Treeck, T., \& Urban, J. (2017). Wirtschaft neu denken: Blinde Flecken in der Lehrbuchökonomie. Berlin: iRights Media.

Open Access Dieses Kapitel wird unter der Creative Commons Namensnennung 4.0 International Lizenz (http://creativecommons.org/licenses/by/4.0/deed.de) veröffentlicht, welche die Nutzung, Vervielfältigung, Bearbeitung, Verbreitung und Wiedergabe in jeglichem Medium und Format erlaubt, sofern Sie den/die ursprünglichen Autor(en) und die Quelle ordnungsgemäß nennen, einen Link zur Creative Commons Lizenz beifügen und angeben, ob Änderungen vorgenommen wurden.

Die in diesem Kapitel enthaltenen Bilder und sonstiges Drittmaterial unterliegen ebenfalls der genannten Creative Commons Lizenz, sofern sich aus der Abbildungslegende nichts anderes ergibt. Sofern das betreffende Material nicht unter der genannten Creative Commons Lizenz steht und die betreffende Handlung nicht nach gesetzlichen Vorschriften erlaubt ist, ist für die oben aufgeführten Weiterverwendungen des Materials die Einwilligung des jeweiligen Rechteinhabers einzuholen.

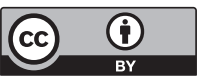

